\title{
O Desafio da Aprendizagem Adaptativa em Programas de Nivelamento para o Ensino Superior
}

\author{
Ismar Frango Silveira, Luis Naito Mendes Bezerra, \\ Carlos Fernando de Araújo Jr., Vilma Silva Lima, Alberto Messias da Costa Souza
}

\author{
Universidade Cruzeiro do Sul \\ Rua Galvão Bueno, 868 - 01506-000 - São Paulo - SP - Brasil \\ \{ismar.silveira, luis.naito, carlos.araujo,vilma.lima, alberto.souza\} @ \\ cruzeirodosul.edu.br
}

\begin{abstract}
Freshmen's placement strategies are recurrently used in the context of Higher Education access, aiming to fill the learning gaps regarding to content, skills and competences, belonging to High School level, which weren't properly developed for any reason. Among these strategies, there are those based on computational solutions, which have the capacities of providing learning contexts adapted to individual students' needs. This aspect represents a real research challenge to be faced. The present paper discusses this challenge, presenting some state-of-the-art elements and bringing some discussions about the topic.
\end{abstract}

Resumo. Estratégias de nivelamento são recorrentemente utilizadas no âmbito do ingresso ao Ensino Superior, no intuito de preencher lacunas de aprendizagem no que diz respeito a conteúdos, habilidades e competências próprias do Ensino Médio, que por diversas razões não são desenvolvidos de maneira adequada. Entre estas estratégias, encontram-se os que se baseiam em soluções computacionais; estas, por sua vez, têm o potencial de propiciar contextos de aprendizagem adaptados às necessidades individuais de cada aluno - o que, por sua vez, é um desafio real de pesquisa a ser explorado. $O$ presente artigo aborda este desafio, apresentando elementos do estado da arte e trazendo discussões sobre esta temática.

\section{Introdução}

Diversos indicadores apontam para deficiências na aprendizagem de Matemática, Linguagem e Ciências no Ensino Médio no Brasil. Resultados do PISA (Programme for International Students Assessment) (OCDE, 2013) mostram que, mesmo tendo havido uma melhora nos resultados a partir de 2003 (ano em que o Brasil iniciou sua participação no exame), os resultados obtidos estão ainda abaixo da média dos países que compõem o exame - o Brasil figura na $58^{a}$ posição entre 65 países. Considerando um indicador interno como o Exame Nacional do Ensino Médio (ENEM), verifica-se que a média nos quatro componentes curriculares orbita em torno de $50 \%$ de aproveitamento (Ciências Humanas: 54,6\%, Ciências da Natureza: 48,2\%, Linguagens: $50,8 \%$, Matemática: 47,3\%). Esse baixo desempenho se reflete, de forma bastante clara, no ingresso dos alunos no Ensino Superior, visto que é notória a dificuldade de grande parcela dos alunos em acompanhar disciplinas que requerem fortemente conceitos prévios oriundos da Educação Básica. Ainda mais grave, há uma parcela significativa desses ingressantes com sérias dificuldades de leitura, escrita e interpretação de texto - 
haja vista que quase $10 \%$ dos que prestaram o ENEM em 2014 tiraram nota zero na Redação (INEP, 2014).

Esse número significativo de ingressantes com lacunas de formação é um dos fatores que impactam nas altas taxas de evasão no Ensino Superior, o que se configura como uma problema internacional - por exemplo, a média de evasão no Ensino Superior nos países europeus fica em torno de $25 \%$, embora a Itália apresente mais de 50\% de evasão (Quinn, 2013). De acordo com Silva Filho et al. (2007), "as perdas de estudantes que iniciam mas não terminam seus cursos são desperdícios sociais, acadêmicos e econômicos".

Desta maneira, tem-se como um desafio de pesquisa em Informática na Educação o estabelecimento de estratégias baseadas em tecnologias que permitam a disponibilização de conteúdos adequados às diferentes lacunas de aprendizagem de cada aluno, com o reaproveitamento de recursos educacionais já disponíveis em cada instituição e repositórios públicos. Para tanto, técnicas de extração automática de metadados podem ser empregadas para otimizar a recuperação de recursos educacionais em repositórios institucionais. Além disso, estratégias inteligentes de recuperação de recursos educacionais em repositórios públicos podem contribuir com o estabelecimento de bases de objetos para a construção de caminhos individualizados de aprendizagem, utilizando técnicas de aprendizagem adaptativa e Educational Data Mining (EDM). Desta maneira, propõe-se neste artigo uma discussão mais aprofundada deste cenário e possibilidades de enfrentamento deste desafio.

O presente artigo encontra-se organizado como segue: a seção 2 discorre sobre o estado da arte no que diz respeito às estratégias e iniciativas de nivelamento na Educação Superior por intermédio de tecnologias; a seção 3 apresenta um panorama das pesquisas atuais sobre aprendizagem adaptativa; a seção 4 traz uma discussão sobre os principais desafios para a aplicação de técnicas de aprendizagem adaptativa em contextos de nivelamento; por fim, apresentam-se as conclusões, trabalhos futuros e referencial bibliográfico.

\section{O estado da arte no nivelamento na Educação Superior por meio de Tecnologias}

De forma a enfrentar a evasão motivada por deficiências de formação, muitas instituições de Ensino Superior vêm implementando programas de nivelamento em conteúdos básicos para alunos ingressantes. Nesses programas, tenta-se resgatar conteúdos básicos, em um movimento justificado pelas políticas de inclusão estimuladas pelo Ministério da Educação. Os programas de nivelamento na realidade da Educação Superior no Brasil em geral se relacionam com o resgate de competências e habilidades que não foram devidamente desenvolvidas em diversas instâncias da Educação Básica, chegando mesmo a resvalar em conceitos pertencentes ao Ensino Fundamental. Em sua maioria, esses programas se desenvolvem através de atividades presenciais, que contam com professores ou monitores seguindo modelos tradicionais de ensino. É possível observar que em países vizinhos, estratégias similares são adotadas pelas universidades, como por exemplo no Chile (PUC Chile, 2014), (UCN, 2014) e Uruguai (ORT, 2015). Lá, como aqui, os programas de nivelamento têm forte ênfase nos conteúdos de Matemática e são voltados para ingressantes nas carreiras de Engenharia e Computação. 
Diversos autores na literatura internacional reportam experiências de cursos de nivelamento por meio de plataformas computacionais, como pode ser visto em Shepherd (2008) e Walker et al. (2011) - ambos apresentam estudos de caso com cursos online para nivelamento de alunos ingressantes no Ensino Superior. Apenas recentemente há registros na literatura sobre o emprego de Massive Online Open Courses (MOOCs) com fins específicos de preencher lacunas de formação, como pode ser visto no trabalho de Kurhila e Vihavainen (2015), por exemplo. Já Kloos et al. (2014) e Muñoz-Merino et al. (2015) relatam o uso de Small Private Open Courses (SPOCs) voltados para ingressantes como vistas à recuperação de conteúdos de Matemática, Física e Química (incluídas no rol de conteúdos conhecidos como STEM Science, Tecnhology, Engineering and Mathematics) do equivalente europeu ao Ensino Médio.

Embora as estratégias de nivelamento citadas tenham seu mérito, há que se reconhecer igualmente suas limitações: tratam-se, em geral, de iniciativas pontuais, que nem sempre alcançam todos os alunos - em especial, as estratégias baseadas em atividades presenciais dificilmente suprem as necessidades do perfil do alunado trabalhador, que não têm flexibilidade de horários para acompanha-las. Nesse sentido, programas que lançam mão de estratégias suportadas por ambientes computacionais poderiam ser uma solução. Porém, tanto os cursos de EAD tradicionais quanto os recentes MOOCs e SPOCs podem não representar uma solução efetiva, dada a sua característica de apresentarem conteúdos e caminhos de aprendizagem pré-definidos, com pouca ou nenhuma flexibilidade, o que pode não ser o adequado para preencher as diferentes lacunas de aprendizagem dos ingressantes, que é o problema em questão. Além disso, todas as estratégias citadas incorrem em custos de mão-de-obra tanto para acompanhamento das atividades presenciais quanto para a elaboração de conteúdos adequados para cada caso, o que pode inviabilizar a implementação dessas iniciativas tanto no setor público quanto no privado.

\section{3. $O$ estado da arte na aprendizagem adaptativa}

No cenário nacional, avanços são encontrados na literatura no que diz respeito à implementação de técnicas de adaptatividade com vistas à aprendizagem personalizada. Exemplos recentes são os trabalhos de Frade et al. (2014), Alves e Jaques (2014), Gomes et al. (2013) e Dorça et al. (2013), utilizando técnicas computacionais distintas que, de maneira geral, baseiam-se em aspectos específicos do modelo adotado (estilos de aprendizagem, conhecimentos prévios, entre outros) para o estudante para obter um certo grau de adaptatividade, o que leva à personalização de determinados aspectos da aprendizagem.

Uma análise da literatura internacional permite verificar uma ênfase na determinação de percursos individualizados (e/ou colaborativos) de aprendizagem (personal learning paths), como pode ser visto em Haack et al. (2010), Ribière et al. (2010), Yang, Hwang e Yang (2013), Santally e Senteni (2013), ou em Ochôa, Carrillo e Cechinel (2014), por exemplo.

Dentro do âmbito da adaptação, de acordo com OET (2010), há três vertentes a se considerar: individualização, personalização e diferenciação. Bray e McClaskey trazem um arrazoado a respeito dessas três dimensões de adaptação, a saber: 
- A individualização diz respeito à definição de objetivos de aprendizagem idênticos para todos os alunos, mas com os progressos obedecendo o ritmo e os conhecimentos prévios de cada estudante;

- A personalização diz respeito à possibilidade de se ter objetivos de aprendizagem ajustados às necessidades, interesses, ritmos e estilos de aprendizagem de cada estudante;

- A diferenciação trabalha com objetivos de aprendizagem idênticos para todos ou para um grupo - de estudantes. O que varia é o método empregado, que deve respeitar as preferências de cada estudante (ou grupos de estudantes com similaridades)

A Tabela 1, a seguir, delineia os elementos principais de cada uma das estratégias.

Tabela 1. Individualização, personalização e diferenciação

\begin{tabular}{|c|c|c|c|}
\hline & Individualização & Personalização & Diferenciação \\
\hline Palavra-chave & "quando?" & "o quê?" & "como?" \\
\hline $\begin{array}{l}\text { Objetivos de } \\
\text { aprendizagem }\end{array}$ & $\begin{array}{l}\text { Todos os estudantes } \\
\text { compartilham dos } \\
\text { mesmos objetivos de } \\
\text { aprendizagem }\end{array}$ & $\begin{array}{l}\text { Cada estudante escolhe } \\
\text { seus objetivos de } \\
\text { aprendizagem }\end{array}$ & $\begin{array}{l}\text { Os objetivos são comuns } \\
\text { para estudantes com } \\
\text { características similares, } \\
\text { podendo ser diferentes } \\
\text { entre grupos distintos de } \\
\text { estudantes }\end{array}$ \\
\hline $\begin{array}{l}\text { Estratégias } \\
\text { didáticas }\end{array}$ & $\begin{array}{l}\text { Aplicação de } \\
\text { diferentes estratégias } \\
\text { didáticas para atingir } \\
\text { os objetivos } \\
\text { educacionais }\end{array}$ & $\begin{array}{l}\text { Aplicação de diferentes } \\
\text { estratégias didáticas para } \\
\text { promover as } \\
\text { potencialidades } \\
\text { individuais }\end{array}$ & $\begin{array}{l}\text { Estratégias livremente } \\
\text { definidas para cada } \\
\text { aluno, podendo utilizar as } \\
\text { mesmas estratégias já } \\
\text { empregadas com alunos } \\
\text { com perfis similares }\end{array}$ \\
\hline Currículo & $\begin{array}{l}\text { Currículo fixo, definido } \\
\text { institucionalmente }\end{array}$ & $\begin{array}{l}\text { O estudante participa } \\
\text { ativamente da } \\
\text { construção de seu } \\
\text { currículo }\end{array}$ & $\begin{array}{l}\text { Currículo flexível: o } \\
\text { currículo pode ser } \\
\text { adaptado aos diferentes } \\
\text { interesses e habilidades } \\
\text { dos estudantes }\end{array}$ \\
\hline $\begin{array}{l}\text { Dimensões do } \\
\text { estudante }\end{array}$ & $\begin{array}{l}\text { Valorização da } \\
\text { dimensão cognitiva do } \\
\text { estudante }\end{array}$ & $\begin{array}{l}\text { Valorização } \\
\text { multidimensional do } \\
\text { estudante (social, } \\
\text { cognitive, afetiva, etc.) }\end{array}$ & $\begin{array}{l}\text { Valorização } \\
\text { multidimensional do } \\
\text { estudante e consideração } \\
\text { dos diferentes estilos de } \\
\text { aprendizagem }\end{array}$ \\
\hline Subsunçores & $\begin{array}{l}\text { Valorização dos } \\
\text { conhecimentos e } \\
\text { habilidades prévias }\end{array}$ & $\begin{array}{l}\text { Valorização dos } \\
\text { conhecimentos, } \\
\text { habilidades e vivências } \\
\text { prévias }\end{array}$ & $\begin{array}{l}\text { Valorização dos } \\
\text { conhecimentos, } \\
\text { habilidades, vivências e } \\
\text { estilos de aprendizagem } \\
\text { previamente } \\
\text { desenvolvidos }\end{array}$ \\
\hline Autonomia & $\begin{array}{l}\text { Autonomia é uma } \\
\text { habilidade secundária }\end{array}$ & $\begin{array}{l}\text { Autonomia é uma } \\
\text { habilidade fundamental }\end{array}$ & $\begin{array}{l}\text { Autonomia é uma } \\
\text { habilidade fundamental }\end{array}$ \\
\hline Papel-chave & Professor & Tutor & Mediador \\
\hline
\end{tabular}


Havendo discutido os elementos que compõem as estratégias de nivelamento no ingresso ao Ensino Superior e o cenário de pesquisa atual no que tange à aprendizagem adaptativa , o item a seguir apresentará os desafios que surgem neste contexto.

\section{Os desafios para a aprendizagem adaptativa em estratégias de nivelamento}

Considerando as discussões dos itens anteriores, quatro dimensões devem ser levadas em considerações em iniciativas de aprendizagem adaptativa: a estruturação curricular definida pelas instituições; a sistematização dos objetivos de aprendizagem - que advêm tanto dos currículos institucionais quanto dos anseios individuais dos estudantes; extração e representação de conhecimentos prévios e lacunas de aprendizagem; e, por fim, a aferição e consideração dos estilos de aprendizagem predominantes nos estudantes (o que pode variar em função do tópico de estudo e de acordo com um conjunto de outros fatores). Uma visão esquemática dessas quatro dimensões e seus relacionamentos é explicitada na Figura 1 a seguir:

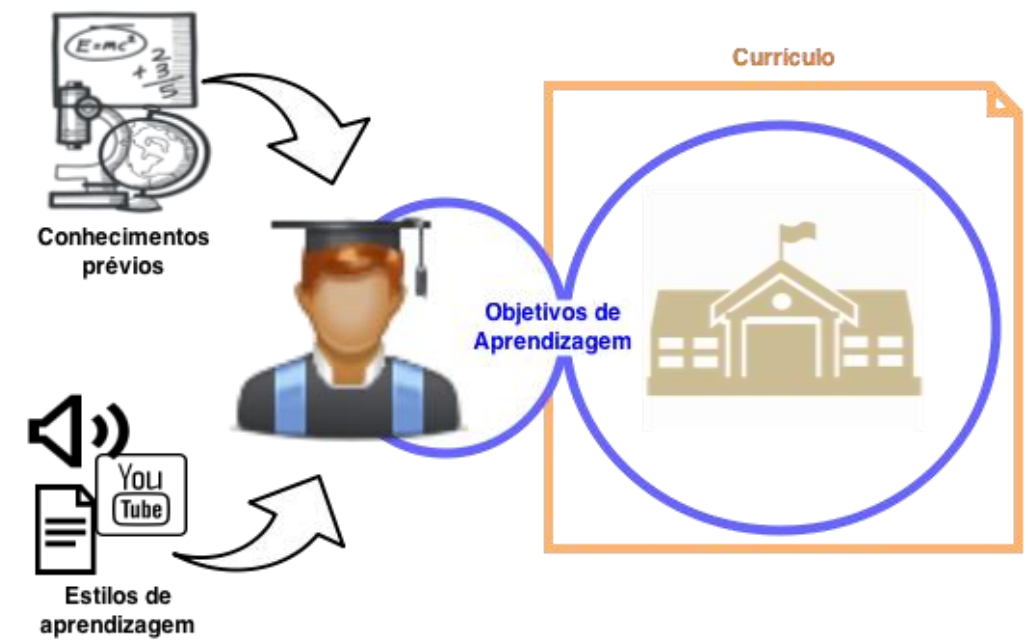

Figura 1 - As quatro dimensões consideradas na aprendizagem adaptativa nos contextos de nivelamento

A compreensão destas quatro dimensões e as possibilidades de utilização de ferramentas e técnicas computacionais que a elas deem suporte são os desafios de pesquisa que serão discutidos neste item.

\subsection{Estruturação e interoperabilidade de Currículos}

A interoperabilidade de currículos, com vistas a processos de certificação e reaproveitamento de estudos, é meta perseguida já há certo tempo. Do ponto de vista governamental, há iniciativas em alguns países, focadas em padronização dos conteúdos, das habilidades e/ou competências. Podem ser citados: os PCN (Parâmetros Curriculares Nacionais) no Brasil (BRASIL, 2000), Common Core Standards para Artes, Linguagem e Matemática (Common Core Standards Initiative, 2015), ou o Framework for K-12 Science Education, na área de Ensino de Ciências (NRC 2012), no âmbito do Ensino Básico dos EUA, ou ainda o Processo de Bolonha na Europa (EC, 
2009), no qual procura-se analisar a compatibilidade curricular entre diferentes países através dos resultados do processo de aprendizagem (learning outcomes), com foco nas competências desenvolvidas.

Entretanto, o real desafio de sistematizar o processo de construção de currículos aparece no sentido de estabelecer estratégias de metapadronização - não de conteúdo, mas de processos. O uso de padrões já estabelecidos, como IEEE LOM (Learning Object Metadata) na especificação de metaestruturas curriculares pode trazer alguns resultados, como aparece no trabalho de Van Assche (2007). Entretanto, a representação de currículos com padrões de metadados requer componentes semânticos para ter efetividade em seu objetivo. Há algumas iniciativas de utilização de ontologias na representação curricular, como no trabalho de Libbretch e Desmoulin (2009), que utilizam de uma representação ontológica para a sub-área de Geometria (Figura 2) - a partir da especificação de uma ontologia de domínio, os autores definem semânticas para competências. Pode-se citar ainda o trabalho seminal de Al-Yahya et al. (2013), que propõem uma ontologia para modelar aspectos de currículo.

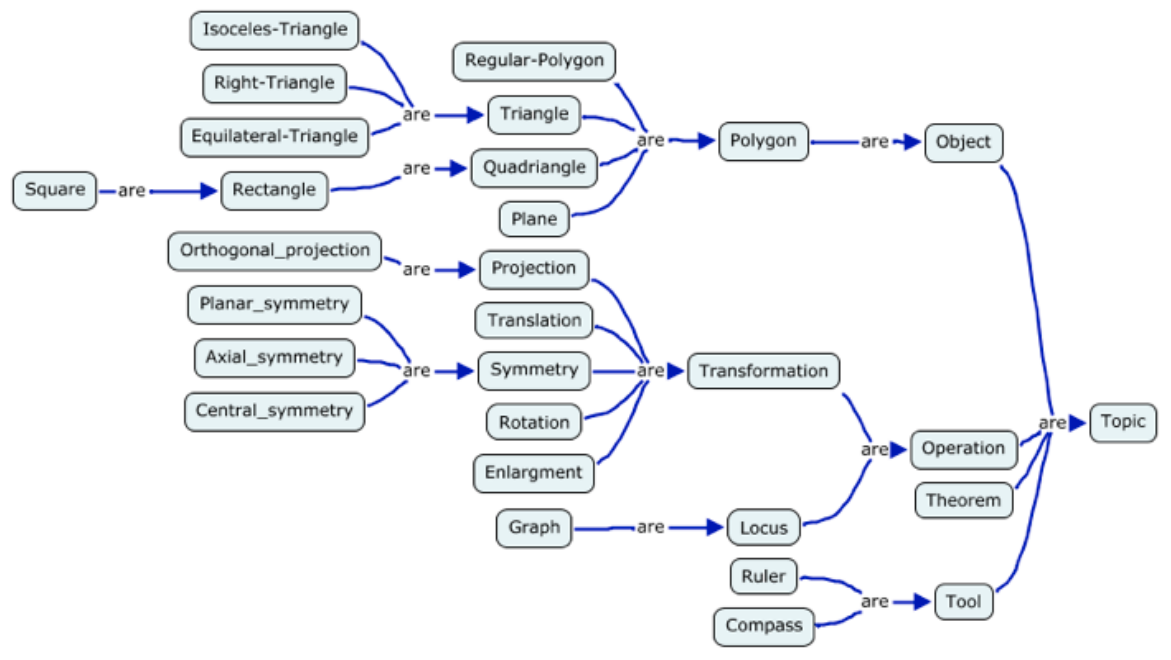

Figura 2 - Exemplo de uso de ontologia para representação curricular [Fonte: Libbretch e Desmoulin (2009)]

\subsection{Sistematização de objetivos de aprendizagem}

Se por um lado os objetivos de aprendizagem são uma parte integrante dos currículos, determinadas estratégias de aprendizagem adaptativa, como a personalização e parte da diferenciação, não se baseiam em estruturas curriculares rígidas, de forma que a definição precisa dos objetivos de aprendizagem depende, em muitos casos, dos interesses individuais dos estudantes. Assim, considerando os objetivos de aprendizagem como partes de dois modelos separados - o modelo do estudante e o modelo do domínio (apropriando-se da clássica terminologia da área de Sistemas Tutores Inteligentes) - tem-se a necessidade de se estabelecer representações semânticas no modelo do estudante que sejam compatíveis com as representações de currículos. Desta maneira, padrões de representação de objetivos de aprendizagem devem ser igualmente buscados.

A consideração de objetivos individuais de aprendizagem, fator essencial para a implementação de técnicas de aprendizagem adaptativa, é fator indispensável para sistemas que possibilitem percursos individualizados de aprendizagem. 


\subsection{Extração e representação de conhecimentos prévios}

A entrada no Ensino Superior é, sem sombra de dúvida, a transição mais impactante no percurso escolar formal, dado principalmente o caráter heterogêneo dos alunos. Os outros momentos de transição (do Ensino Fundamental para o Médio e da Graduação para a Pós-Graduação) beneficiam-se de uma menor heterogeneidade de formação dos alunos, bem como de uma maior possibilidade de trabalhar currículos como um processo contínuo. A formação generalista do Ensino Médio (excetuando-se, claramente, os cursos de formação profissionalizante) cria lacunas de aprendizagem, ao não trabalhar com a ênfase necessária em tópicos que são pré-requisitos das carreiras do Ensino Superior.

Aqui, a estruturação e a interoperabilidade curriculares desempenham papéis fundamentais: a definição de estruturas para mapeamento de coberturas conceituais - de mapas conceituais a ontologias (Libbretch e Desmoulins, 2009; Shitsuka et al., 2011; Ochôa et al., 2014) - pode auxiliar a construção de sistemas de avaliação diagnóstica que determinem as lacunas de aprendizagem individuais, ao estabelecer os prérequisitos conceituais do Ensino Médio para o desenvolvimento cognitivo adequado das competências e habilidades de cada carreira do Ensino Superior.

\subsection{Aferição de estilos de aprendizagem}

Apesar de todo o criticismo que há tempos cerca as pesquisas em Estilos de Aprendizagem - vide os trabalhos de Curry (1990) até Coffield et. al. (2004), por exemplo - os mesmos não podem - ou não deveriam ser - ignorados, em especial quando se trata de elaboração, definição e sequenciamento (automático ou não) de recursos de aprendizagem.

Uma análise da literatura permite considerar a ainda prevalência dos modelos clássicos de categorização de Estilos, como os de Felder e Silverman (1988) e Kolb (1984), com a utilização de questionários como principal instrumento para aferição dos estilos. Um desafio de pesquisa ainda não suplantado é a evolução de estratégias efetivas de aferição automática de Estilos de Aprendizagem, como nos trabalhos de Graf et al. (2009) e Özpolat e Akar (2009)

\section{Conclusões e Trabalhos Futuros}

O presente artigo propôs-se a trazer uma discussão acerca dos desafios de pesquisa para a comunidade de Informática na Educação no que diz respeito às estratégias computacionais de aprendizagem adaptativa no contexto do nivelamento para o Ensino Superior. Foram destacados aspectos relativos ao próprio conceito de adaptação (que envolve individualização, personalização e diferenciação), à luz de uma análise da evolução do estado da arte quanto aos mecanismos que permitem certos graus de adaptatividade. No contexto específico da necessidade de nivelamento para ingressantes no Ensino Superior, ressaltou-se a heterogeneidade como um aspecto determinante, o que requer a adoção de estratégias que permitam a estruturação e interoperabilidade curricular, a sistematização dos objetivos de aprendizagem, a extração dos conhecimentos prévios e a aferição de estilos de aprendizagem.

Tendências futuras de pesquisa para os próximos anos apontam para a integração, neste cenário, de elementos relativamente recentes no contexto educacional, como ferramentas de Educational Data Mining (EDM) e Learning Analytics. Neste 
âmbito, o uso mais sistematizado de dados gerados de forma massiva por ambientes formais e não-formais - de aprendizagem, aliado a um aprimoramento dos algoritmos de manipulação e análise desses dados, pode ser um caminho que auxilie políticas de sucesso por parte de IES no acolhimento e integração de seus novos estudantes.

\section{Referências}

Alves, F. P. e Jaques, P. (2014). Um Ambiente Virtual com Feedback Personalizado para Apoio a Disciplinas de Programação. In: Anais do XXV Simpósio Brasileiro de Informática na Educação - SBIE 2014, p. 1078-1082.

BRASIL. MINISTÉRIO DA EDUCAÇÃO (2000). Parâmetros Curriculares Nacionais para o Ensino Médio. Brasília: MEC.

Bray, B. e McClaskey, K. (2010) Personalization vc Differentiation vs Individualization. White paper. Disponível na Internet em < https://ideas.education.alberta.ca/media/81484/personalizationvsdifferentiationvsindivid ualization.pdf $>$. Acesso em 11 de maio de 2015.

Coffield, F.; Moseley, D.; Hall, E.; Ecclestone, K. (2004) Should we be using Learning Styles? What research has to say to practice. London: Learning and Skills Research Centre.

Common Core Standards Initiative (2015). Common Core Stardards for Mathemticis. Disponível na Internet em: < http://www.corestandards.org/wpcontent/uploads/Math_Standards.pdf $>$. Acesso em 14 de maio de 2015.

Curry, L. (1990) A Critique of the Research on Learning Styles. In: Educational Leadership, p. 50-56.

Dorça, F. et al. (2013). Uma Abordagem para Geração Automática de Conteúdo Personalizado Através da Recomendação Estocástica de Objetos de Aprendizagem no Processo de Ensino em Sistemas Adaptativos e Inteligentes para Educação. In: Anais do XXIV Simpósio Brasileiro de Informática na Educação - SBIE 2013, p. 838-848.

EC - European Communities (2009) ECTS Users' Guide. Luxemburgo: Office for Official Publications of the European Communities.

Felder, R. M.; Silverman, L. (1988) Learning and Teaching Styles. In: Engineering Education, 78(7), p.674-681.

Frade, R. V. C. et al. (2014). Um Ambiente Virtual 3D Multiagente com Recomendação Personalizada de Objetos de Aprendizagem. In: Anais do XXV Simpósio Brasileiro de Informática na Educação - SBIE 2014, p. 1068-1077.

Graf, S.; Kinshuk; Liu, T.-C. (2009) Supporting Teachers in Identifying Students' Learning Styles in Learning Management Systems: An Automatic Student Modelling Approach. In: Learning Management Systems: An Automatic Student Modelling Approach. Educational Technology \& Society, 12 (4), p. 3-14.

Haack, B.; Koppatz, P.; Scholl, M.; Sistenich, F.; Tippe, U. (2010) E-Learning and Further Education: How do Individual Learning Paths support Personal Learning Processes. In: Systemics, Cybernetics and Informatics, v. 8, p. 75-79. 
Gomes, E. H.; Pimentel, E. P.; Omar, N.; Marietto, M. G. B. (2013) Personalização do E-Learning Baseado no Nível de Aquisição de Conhecimentos do Aprendiz. In: Anais do XXIV Simpósio Brasileiro de Informática na Educação - SBIE 2013, p. 637-646.

Kloos, C. D. et al. (2014) Experiences of running MOOCs and SPOCs at UC3M. In: Proceedings of the 2014 IEEE Global Engineering Education Conference - EDUCON. Istambul, Turquia, p. 884-891.

Kolb, D. A. (1984) Experiential learning: experience as the source of learning and development. Englewood Cliffs, NJ: Prentice Hall.

Kurhila, J. e Vihavainen, A. (2015) A Purposeful MOOC to Alleviate Insufficient CS Education in Finnish Schools. In: ACM Transactions on Computing Education, v. 15, 2, maio, artigo 10.

Libbrecht, P. e Desmoulin, C. (2009) A Cross-Curriculum Representation for Handling and Searching Dynamic Geometry Competencies. In: Dicheva, D., Mizoguchi, R. e Greer, J. (eds) Semantic Web Technologies for e-Learning, Volume 4 - The Future of Learning, p. 136-158.

Muñoz-Merino, P. J. et al. (2015) Precise Effectiveness Strategy for analyzing the effectiveness of students with educational resources and activities in MOOCs. In: Computers in Human Behavior, v. 47, p. 108-118

NRC - National Research Council (2012). A Framework for K-12 Science Education: Practices, Crosscutting Concepts, and Core Ideas. Washington, DC, EUA: The National Academies Press.

Ochôa, X.; Carrillo, G.; Cechinel, C. (2014) Use of a Semantic Learning Repository to Facilitate the Creation of Modern e-Learning Systems. In: Proceedings of Interacción 2014. Tenerife, Espanha.

OECD (2008) Education at a Glance. Disponível na Internet em <http://www.oecd.org/education/skills-beyond-school/41284038.pdf>. Acesso em 7 de maior de 2015.

OECD (2014) PISA 2012 Results in Focus - What 15-year-olds know and what they can do with what they know. Disponível na Internet em <http://www.oecd.org/pisa/ keyfindings/pisa-2012-results-overview.pdf>. Acesso em 8 de abril de 2015

OET (2010) National Education Technology Plan 2010. Disponível na Internet em < http://tech.ed.gov/wp-content/uploads/2013/10/netp2010.pdf $>$. Acesso em 11 de abril de 2015.

ORT Universidad (2015) Nivelación em Matemática. Disponível na Internet em <http://fi.ort.edu.uy/nivelacion-en-matematica>. Acesso em 10 de abril de 2015.

Özpolat, E. e Akar, G. D. (2009) Automatic detection of learning styles for an elearning system. In: Computers \& Education, v. 53, p. 355-367.

PUC Chile. Con buenos resultados terminó el curso de nivelación en Precálculo. Disponível na Internet em <https://www.ing.puc.cl/con-buenos-resultados-termino-elcurso-de-nivelacion-en-precalculo/>. Acesso em 10 de abril de 2015. 
Quinn, J (2013) Drop-out and Completion in Higher Education in Europe. Disponível na Internet em <http://www.nesetweb.eu/sites/default/files/HE\%20Drop\%20out\%20 AR\%20Final.pdf $>$. Acesso em 8 de maio de 2015.

Ribière, M.; Picault, J.; Squedin, S. (2010) The sBook: Towards Social and Personalized Learning Experiences. In: Proceedings of BooksOnline'10. Toronto, Canada.

Santally, M. I. e Senteni, A. (2013) Effectiveness of Personalised Learning Paths on Students Learning Experiences in an e-Learning Environment. In: European Journal of Open, Distance and E-Learning, v. 16 (1), p. 36-52.

Shepherd, C. M. (2008) Any time, any place: online advanced placement courses for high school students. In: Journal of Universal Access in the Information Society, v. 7 (4), outubro, p. 285-292.

Shitsuka, R.; Silveira, I. F.; Shitskuta, D. M. (2011) Comparação entre as ferramentas Ontologia, Mapas Mentais e Mapas Conceituais na representação de conceitos em matriz curricular de curso de graduação. In: Revista CRB-8 Digital, São Paulo, v. 4, n. 1, p. 2-10.

Silva Filho, R. L. B; Motejunas, P. R.; Hipólito, O.; Lobo, M. B. C. M. (2007) A evasão no ensino superior brasileiro. In: Cadernos de pesquisa, São Paulo, v. 37 (132), p. 641659.

UCN - Universidad Católica del Norte (2015). Estudiantes que ingresen a Ingeniería recibirán nivelación gratuita. Disponível na Internet em $<$ http://www.noticias.ucn.cl/destacado/estudiantes-que-ingresen-a-ingenieria-recibirannivelacion-gratuita/> . Acesso em 10 de abril de 2015.

Van Assche, F. (2007) Linking Learning Resources to Curricula by using Competencies; In: Proceedings of the First International Workshop on Learning Object Discovery \& Exchange. Creta, Grécia, p. 80-91.

Walker, H. M.; Hirakawa, A.; Steinbach, R. (2011) A system to place incoming students in computer science, mathematics and statistics. In: Journal of Computing Sciences in Colleges, v. 27 (1), p. 24-31.

Yang, T.-C.; Hwang, G.-J.; Yang, S. J.-H. (2013). Development of an adaptive learning system with multiple perspectives based on students' learning styles and cognitive styles. In: Educational Technology \& Society, 16 (4), p. 185-200. 